\title{
Next-generation sequencing reveals sterile crustose lichen phylogeny
}

\author{
Hodkinson $\mathrm{BP}^{1,2}$ and Lendemer $\mathrm{JC}^{2}$ \\ ${ }^{1}$ Perelman School of Medicine, University of Pennsylvania, BRB 1046A, 421 Curie Blvd, Philadelphia, PA 19104 \\ ${ }^{2}$ Institute of Systematic Botany, The New York Botanical Garden, Bronx, NY 10458-5126
}

Hodkinson BP, Lendemer JC 2013 - Next-generation sequencing reveals sterile crustose lichen phylogeny. Mycosphere 4(6), 1028-1039, Doi 10.5943/mycosphere/4/6/1

\begin{abstract}
The rapid phylogenetic placement and molecular barcoding of fungi is often hampered in organisms that cannot easily be grown in axenic culture or manually teased apart from their associated microbial communities. A high-throughput procedure is outlined here for this purpose, and its effectiveness is demonstrated on a representative species from an especially problematic group of fungi, the sterile crustose lichens. Sequence data of the LSU and ITS regions were generated from samples of a sterile crustose lichen species, Lepraria moroziana, using nextgeneration sequencing. DNA fragments most likely to represent the primary lichen-forming fungus were bioinformatically teased out using a specialized data processing pipeline. Phylogenetic analyses of the LSU region revealed that the lichen-forming fungus L. moroziana was previously placed in the incorrect class of fungi (Lecanoromycetes), and actually belongs to the class Arthoniomycetes, in the order Arthoniales. It is here treated as a member of a new family (Andreiomycetaceae Hodkinson \& Lendemer fam. nov.) and genus (Andreiomyces Hodkinson \& Lendemer gen. nov.). Additionally, Lepraria obtusatica Tønsberg is placed in the newly-defined genus based on its morphological, chemical, and ITS-based molecular similarity to L. moroziana. The procedure outlined here is projected to be especially useful for resolving the dispositions of diverse problematic fungi that remain unnamed, incertae sedis, or have taxonomic positions that are not expected to reflect their true phylogeny.
\end{abstract}

Key words - Andreiomyces - Andreiomyces morozianus - Andreiomycetaceae - Arthoniales Arthoniomycetes - Ascomycota - Ascomycetes - fungal barcoding - Lepraria - Lepraria moroziana - ITS - LSU - nrITS - nrLSU - nucITS - nucLSU - microbiome - mothur - Roche 454 - sequencing contamination - taxonomy - uncultured fungi

\section{Introduction}

In recent years, next-generation sequencing has revolutionized the fields of genetics and genomics. However, with the exception of notable recent 'phylogenomic' studies (Roeding et al. 2009, Smith et al. 2011, Timme et al. 2012), most systematists studying macroscopic organisms currently generate the bulk of molecular sequence data through Sanger sequencing (e.g. Lendemer \& Hodkinson 2009, 2010, Hodkinson et al. 2010, Hodkinson \& Lendemer 2011, Schmull et al. 2011, Gryganskyi et al. 2012). While this approach works well for some groups of organisms, there are others that are plagued by problems of contamination. These problems are typically circumvented through the process of molecular cloning, which adds significant extra cost and effort to the data-generation process. 
A Sanger sequence, by definition, represents a summary of the amplicons found in a pool. If the pool is derived from a PCR reaction performed on a sample with multiple organisms, there is the potential that such a summary would not result in a readable sequence, or that a sequence from another organism (that is not the organism of interest) would dominate the pool (Taranger et al. 1994, Hofstetter et al. 2007). With next-generation amplicon sequencing and Sanger sequencing of cloned amplicons, each sequence is presumably derived from a single original DNA fragment (Margulies et al. 2005, Edwards et al. 2006, Ley et al. 2006, Turnbaugh et al. 2006, 2007, Liu et al. 2007, Roesch et al. 2007). These approaches, therefore, allow one to examine the sequences of individual amplicons and use bioinformatics to determine which sequence belongs to the organism(s) of interest.

The problems associated with using Sanger sequencing to generate a single sequence of one gene from one sample are especially pronounced in the highly speciose assemblage of lichens known as sterile asexually-reproducing crustose lichens, or 'sterile crusts' (Hodkinson \& Lendemer 2012a, 2012b). Lichen-forming fungi typically live alongside numerous fungal and bacterial associates and create microcosms in which these diverse communities develop (Hodkinson \& Lutzoni 2009). Since lichen-forming fungi are typically extremely resistant to culture, samples of these organisms are almost always comprised of the many diverse organisms that inhabit the lichen microbiome (Hodkinson 2011, Hodkinson et al. 2012a). It is important to note that the 'sterile crusts' do not represent a monophyletic group, but rather are treated together because they have all evolved to reproduce primarily through the dispersal of lichenized diaspores (specialized dispersal units that include the major constituents of the lichen microbiome) (Hodkinson \& Lendemer 2012c). Species that employ this mode of reproduction have evolved in nearly all of the diverse lineages that comprise lichen-forming fungi (Lendemer \& Hodkinson 2012a, 2013). However, since they typically do not display any sexual state, they frequently do not have the morphological characters that can be used to determine their higher-level phylogenetic placement (Lendemer \& Hodkinson 2012b). As a result of both their phylogenetic diversity and lack of characters that inform higher-level placement, one often cannot a priori determine the major fungal lineage to which a particular species belongs (Hodkinson \& Lendemer 2012c, Lendemer \& Hodkinson 2013). This makes it nearly impossible to successfully and efficiently design primers that would be specific enough to reduce contamination of the amplicon pool with sequences from endolichenic fungi. For these reasons, fungal contamination remains a major issue when studying sterile crustose lichens, and Sanger sequencing of amplicon pools derived directly from lichen samples frequently fails to produce usable results (Lendemer 2012).

In an effort to phylogenetically place sterile crustose lichen species in the fungal tree of life, we have developed a set of high-throughput, cost-effective (as compared to cloning) procedures to generate and process sequence data from specific, well-studied loci (LSU and ITS) using nextgeneration (Roche 454) sequencing. We demonstrate the effectiveness of this procedure using a single species for which the generation of DNA sequence data has proven to be difficult when using Sanger sequencing. Our results indicate that its taxonomic placement was incorrect at the class, order, family, and genus level, and we establish a new family and genus to accommodate it. Further, non-molecular character and ITS sequence data are used to place an additional species in the new genus. We discuss implications for the 'barcode of life' effort and the utility of this method for placing a diverse array of poorly-studied, unculturable organisms.

\section{Materials \& Methods}

\section{Non-molecular materials and methods}

Chemical and morphological analyses of specimens representing the lichen-forming fungi Lepraria moroziana Lendemer and L. obtusatica Tønsberg were carried out using the methodology outlined in detail by Lendemer et al. (2008) with the SEM methods as corrected by Lendemer and Elix (2010). Descriptive morphological terminology follows Lendemer (2011). 
Table 1 - Samples for which ITS and LSU sequence data were collected for this study. All vouchers are stored at the New York Botanical Garden (NY).

\begin{tabular}{|c|c|c|c|c|c|c|}
\hline Species & Collector & State/Province & Coll. \# & DNA ID & LSU GB Acc. & ITS GB Acc. \\
\hline \multirow{10}{*}{ Andreiomyces morozianus } & Lendemer & Pennsylvania, USA & 25680 & NY0976 & KF740833 & - \\
\hline & & & 25675 & NY0978 & KF740832 & - \\
\hline & & & 25681 & NY0979 & KF740831 & - \\
\hline & & & 25677 & NY0982 & KF740830 & - \\
\hline & & New Brunswick, CA & $27771 \mathrm{~A}$ & NY1183 & KF740829 & - \\
\hline & & North Carolina, USA & 29707 & NY1196 & KF740828 & KF740836 \\
\hline & & Tennessee, USA & 29836 & NY1223 & KF740827 & KF740837 \\
\hline & & & 29809 & NY1227 & KF740826 & KF740838 \\
\hline & & & 30380 & NY1373 & KF740834 & KF740839 \\
\hline & & & 30439 & NY1377 & KF740835 & KF740840 \\
\hline
\end{tabular}

\section{DNA extraction and Sanger sequencing}

DNA extractions were performed on 10 subsamples of Lepraria moroziana specimens (Table 1) as outlined by Lendemer \& Hodkinson (2013) using the DNeasy Plant Mini Kit (Qiagen) with the instructions modified to include a prolonged ( 12 hour) incubation period in lysis buffer. Isolated DNA was resuspended in $100 \mu \mathrm{l}$ of sterile water and stored at $-20^{\circ} \mathrm{C}$. ITS sequence data were generated as outlined by Hodkinson and Lendemer (2011). [The above steps were performed by the second author (JCL) at NYBG; the remaining steps outlined below were carried out by the first author (BPH) at NYBG.] MegaBLAST searches of the resulting sequences were performed against the NCBI non-redundant nucleotide collection to screen out contaminant and endolichenic fungal sequences (Zhang et al. 2000).

\section{Roche 454 sequence data collection}

PCR amplifications on the 10 aforementioned DNA extracts (Table 1) were performed using the primer combinations ITS1f/ITS4 (White et al. 1990, Gardes \& Bruns 1993) and LROR/LR3 (Vilgalys \& Hester 1990, Rehner and Samuels) with barcoded LibL 'A' adapters appended to the forward primers (for list of barcode sequences, see the '.oligos' files in the associated data package; http://sites.google.com/site/brendanhodkinson/) and LibL 'B' adapters appended to the reverse primers. Each barcode was associated with a single sample, as indicated in the aforementioned '.oligos' files, with the same barcode always being used for both the ITS and LSU amplicons of each sample. Amplification reactions of $50 \mu 1$ were prepared as outlined in the Green Taq DNA Polymerase Technical Manual (No. TM0586; GenScript, Piscataway, NJ) containing $2 \mu \mathrm{l}$ of each primer (in solution at a concentration of $10 \mu \mathrm{M}$ ) and $2 \mu 1$ of extracted DNA. Thermocycler settings followed Hodkinson \& Lutzoni (2009, see also Hodkinson, 2011). PCR products were studied prior to sequencing by running $2 \mu \mathrm{l}$ of each amplified PCR product on a $1 \%$ agarose gel using electrophoresis, staining the gel with ethidium bromide, and visualizing the gel under UV light. Samples were then pooled with 86 additional samples representing an assortment of diverse sterile crustose lichen species that had been prepared in the same manner using equal amounts of each PCR product. Pooled, unpurified PCR products were sent to the Duke University Genome Sequencing \& Analysis Core Resource for sequencing. Small fragment removal was performed according to Roche protocols using AMPure XP beads (Beckman Coulter Genomics). The final pool was run on a High Sensitivity DNA Chip (Agilent Tech.). The samples were then run on one eighth of a Roche 454 GS-FLX PTP plate with Titanium series reagents, sequencing from the 'A' adaptor only.

\section{Next-generation sequence data processing}

An automated pipeline for sequence data processing was written as a batch file for the program Mothur 1.25 (Schloss et al. 2009). The pipeline was designed to: (1) perform initial processing of Roche 454 ITS and LSU amplicon data; (2) identify and segregate sequences from Lecanoromycetes and Arthoniomycetes; (3) identify and exclude chimeras; (4) cluster sequences and identify centroid sequences for each cluster, and (5) identify and exclude centroid sequences 
from singleton-based clusters. The following reference files are used for classifying sequences and determining which are potentially chimeric: (1) fungalITSdatabaseID.fasta (a sequence reference

file for ITS classification and chimera checking, available at http://www.emerencia.org/chimerachecker.html), (2) ITS_Database_Hodkinson.tax (a taxonomy reference file for ITS classification available in the associated data package; it is a manually corrected version of the taxonomy file that goes with the previous FASTA file), (3) FungiLSU_train_1400bp_8506_mod.fasta (a sequence reference file for LSU classification; found at http://www.mothur.org/wiki/RDP_reference_files), (4) FungiLSU_train_1400bp_8506_mod.tax (taxonomy reference file for LSU classification associated with the previous FASTA file), and (5) james.trim.mafft (an alignment of LSU reference sequences for chimera checking created by downloading the alignment assembled by James et al. (2006) from the AFTOL website, removing non-LSU portions, taking out taxa that are non-fungal or missing LSU, and re-aligning with MAFFT (Anthony Amend, pers. comm.)). A Perl script (ITS_database_fasta_seqID_renamer.pl; available in the associated data package) is also called as part of the pipeline to reformat the fungal ITS sequence database ('fungalITSdatabaseID.fasta') for use by Mothur. At each step in the pipeline, relevant files for each gene/sample combination are output in FASTA format with a corresponding quality file. UCHIME (Edgar et al. 2011) and USEARCH (Edgar 2010) are invoked as part of the pipeline to identify potential chimeras and centroid sequences of clusters, respectively. Specific cutoffs for determining (1) read length/quality, (2) taxonomic assignment, (3) and clustering (which impacts how centroids and singletons are defined) can be found in the main Mothur batch file (http://sites.google.com/site/brendanhodkinson/).

Some samples in the full set of 96 did not produce ITS sequences that could be classified with confidence in either Lecanoromycetes and Arthoniomycetes, probably due to the variable nature of ITS, the frequency of introns, and the lack of close relatives in the ITS sequence database. A special partial pipeline was designed for these samples to perform the functions outlined above, with the exception of the functions associated with excluding sequences based on taxonomy. This 'taxonomy free' ITS sequence processing pipeline is available as a Mothur batch file in the associated data package.

\section{Phylogenetic analyses}

i. Initial phylogenetic mapping - In order to ascertain the placement of the sampled sterile crustose lichen-forming fungi in the Ascomycota, we used RAxML 7.2.6 (Stamatakis 2006) to map the sequences newly generated for this study onto the Ascomycota phylogeny generated by Schoch et al. (2009). First, the nucleotide alignment associated with that study was downloaded from TreeBASE (http://treebase.org/). Using Mesquite 2.74 (Maddison \& Maddison 2010), all non-LSU sites were manually pruned out of the alignment, along with taxa not represented by sequences from the LSU region. The LSU sequences generated for this study were then aligned manually with those in the pruned dataset. Reference sequences that did not overlap with the shortest of the newly-generated sequences were deleted. Ambiguously aligned regions were then excluded by defining them as part of an exclusion set and the alignment was saved in NEXUS format. The ambiguously aligned regions were then manually deleted and the alignment exported in extended PHYLIP format for use with RAxML. The topology included in the downloaded alignment file generated by Schoch et al. (2009) was then used as a reference tree for mapping the newly generated sequences. The topology had been automatically modified by Mesquite to remove taxa from the tree when they were deleted as part of editing the alignment (above). The pruned tree was saved as a separate file in standard NEWICK format for use with RAxML.

After completing the above, the alignment and the reference tree were used as input files for RAxML and the "-f y" command was invoked to map the sequences onto the tree. The results of this procedure were visualized with FigTree 1.3.1 (Rambaut 2009) and used to inform subsequent phylogenetic analyses. 


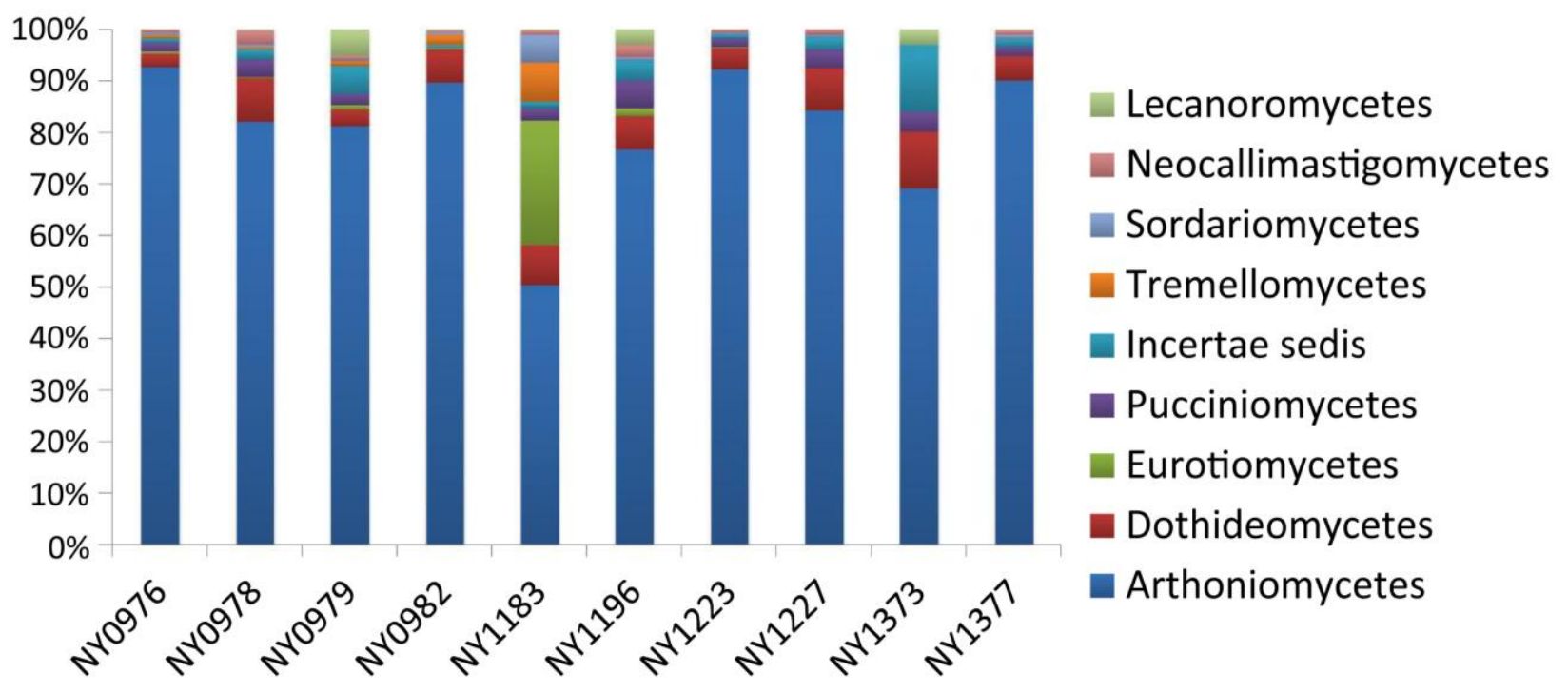

Fig. 1 - Relative abundances of the various putative classes represented by LSU sequences in each of the ten Andreiomyces morozianus samples.

ii. Arthoniales phylogenetic analyses - All samples of the lichen-forming fungus Lepraria moroziana produced sequences that were phylogenetically mapped as sister to the Arthoniales clade in the previous analysis. To test the hypothesis that this fungus may represent one of the more basal lineages within Arthoniales, the sequences from this clade were combined with the LSU sequences from the Ertz and Tehler (2011) Arthoniales data set. Sequence identifiers were copied in batches from the Ertz and Tehler (2011) supplement and pasted into a file in the '.accnos' format (http://www.mothur.org/wiki/List.seqs). The full text of the 'accnos' file was copied and pasted into the search box on the NCBI nucleotide core site (http://www.ncbi.nlm.nih.gov/nuccore/). The search results were downloaded in FASTA format and sequence identifiers were reconfigured using a customized Perl script modified from sequence_renamer.pl script published by Hodkinson et al. (2012b, 2012c) (available in the supplementary information). Sequences from Lepraria moroziana that were phylogenetically mapped as sister to the Arthoniales clade in the previous analysis were added to the FASTA file and MAFFT 6.956 (Katoh et al. 2002) was used to create a provisional alignment, which was realigned manually using Mesquite. Ambiguously aligned regions were excluded by defining them as part of an exclusion set and the alignment was saved in NEXUS format. The ambiguously aligned regions were then manually deleted and the alignment exported in extended PHYLIP format. Maximum likelihood (ML) phylogenetic analyses were run with RAxML using the "-f a" command (500 bootstrap replicates followed by a thorough topology search). One set of analyses was run without a topological constraint, while another was run with a backbone constraint tree matching the one shown by Ertz. et al. (2011) with the following exceptions: (1) taxa representing duplicate sequences were removed and (2) all family-level clades were left as polytomies. RAxML was used to map the bootstrap proportions from the unconstrained bootstrap analysis to the best scoring topology inferred with a backbone constraint (using "-f b"). Results were visualized with FigTree 1.3.1.

\section{Results}

For every Lepraria moroziana LSU amplicon pool, the majority of sequences were identified as 'Arthoniomycetes,' with the remainder being split between various fungal classes (Fig. 1). The corresponding Roche 454-generated ITS results had only four of the ten samples having a majority of sequences identified as belonging to a single fungal class (NY0976 - 69\% 'Saccharomycetes'; NY0978 - 53\% 'Saccharomycetes'; NY0979 - 63\% 'Lecanoromycetes'; NY0982 - 57\% 'Saccharomycetes'); the remaining six samples had no class in the majority. The only class-level identifications associated with ITS sequences in all samples were 


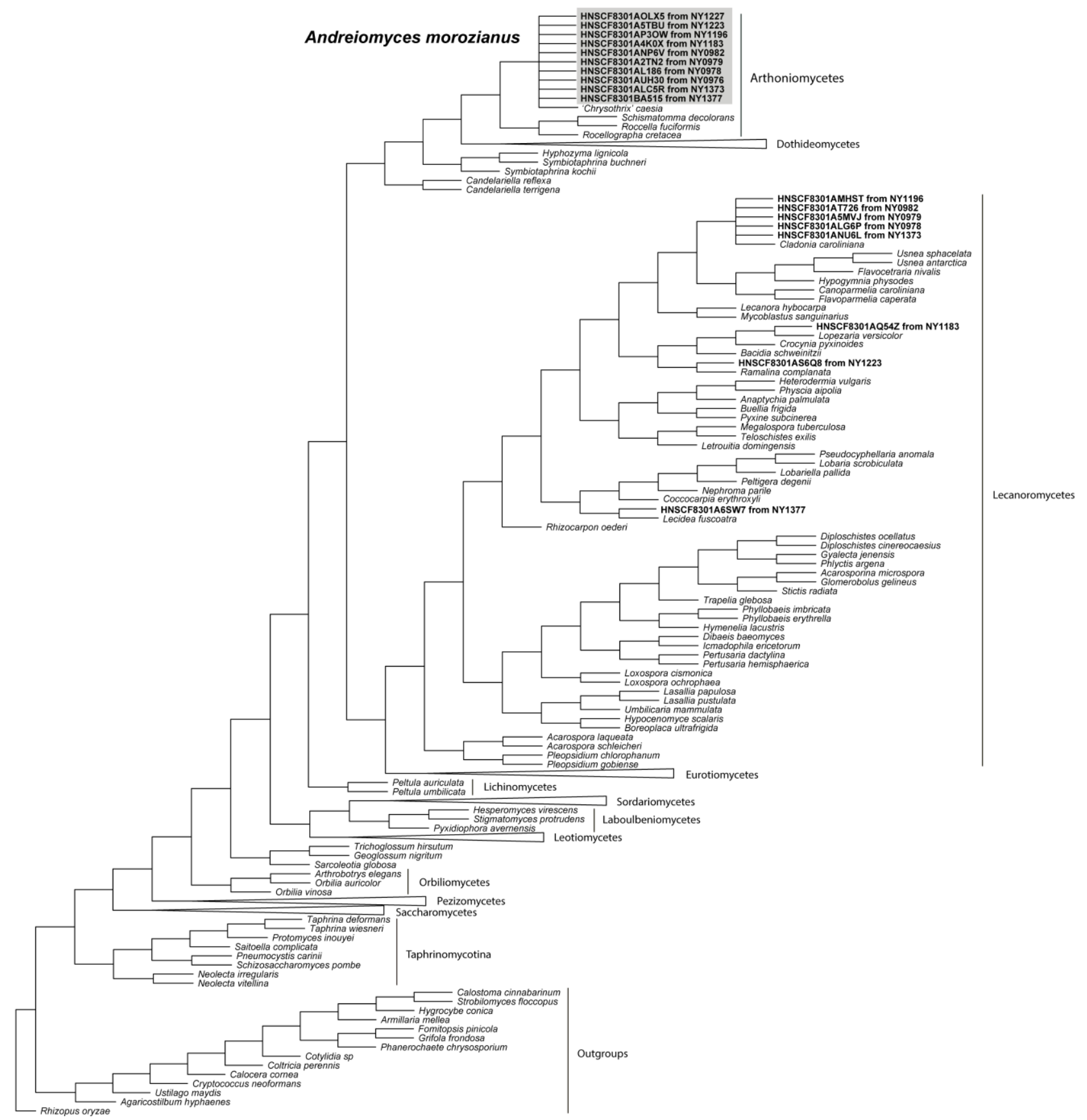

Fig. 2 - Ascomycota cladogram with all Arthoniomycete and Lecanoromycete sequences from the ten Andreiomyces morozianus samples (in bold) mapped using the RAxML Evolutionary Placement Algorithm (EPA).

'Saccharomycetes' and 'Dothideomycetes' (see associated data package for a detailed taxonomic identification profile). OTU-based analyses showed that there was a single ITS OTU that was present in all samples, and it was the second most abundant in the data set overall (the overall most abundant OTU was found in only three samples; see associated data package for OTU table).

Phylogenetic mapping of the final cluster centroid LSU sequences from Lepraria moroziana samples demonstrated that each amplicon pool had a sequence that went into a single group mapped within Arthoniales (closest to 'Chrysothrix'/'Arthonia' caesia) (Fig. 2). Sequences from Lecanoromycetes were also found within the samples, although no sequence group from that class was found consistently in all samples. Our LSU-based phylogenetic analyses of Arthoniales showed Lepraria moroziana to be in a well-supported position sister to the sequences representing Chrysotricaceae (Fig. 3). 


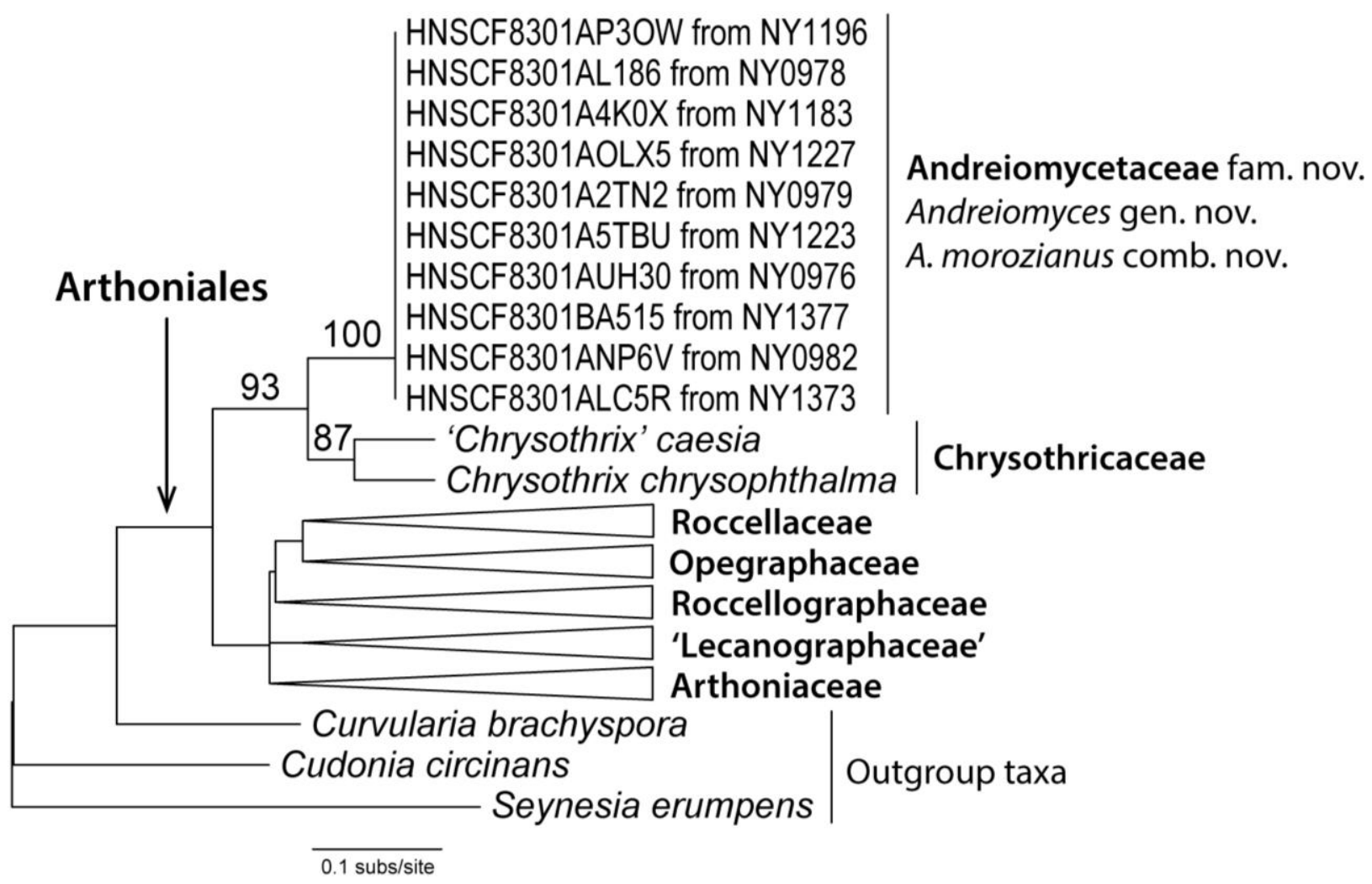

Fig. 3 - Phylogenetic reconstruction of Arthoniales highlighting the new family and genus. The topology was inferred with maximum likelihood (ML) using a backbone constraint (as described in the Materials and Methods). ML-BP support values were inferred without constraints and values $\leq 50$ are not shown.

Five of the ten samples yielded ITS sequences through Sanger sequencing (NY1196, NY1223, NY1227, NY1373, and NY1377). The highest BLAST hit for each one was Lepraria obtusatica (AF517896; E-value range: 2e-63 to 6e-144; bitscore range: 252 to 520), with Chrysothrix sp. (AB764067) being the second highest hit (E-value range: 3e-22 to 1e-76; bitscore range: 115 to 296). An intron $\sim 0.5 \mathrm{~kb}$ in size was sequenced at the 5 ' end of the forward read (although for most of the samples, contaminating sequences masked this portion of the fragment).

\section{Discussion}

The results of our study indicate that the species in question, Lepraria moroziana, has been misclassified at the class, order, family, and genus level due to its morphological similarity to members of the genus Lepraria s. str. In accordance with phylogenetic analyses, we place the species in the order Arthoniales (class Arthoniomycetes), and establish a new family (Andreiomycetaceae) and genus (Andreiomyces) to accommodate it. Based on non-molecular characters and ITS sequence data, we combine an additional species, Lepraria obtusatica Tønsberg, into the new genus.

All samples had a majority of sequences representing Arthoniales based on 454-generated LSU sequence data. Together, these reads formed a tight cluster of highly similar sequences, leading us to conclude that the main lichen-forming fungus belonged to this order. The various other sequences from different parts of the fungal tree can be explained by the presence of endolichenic fungi and/or the close proximity of sampled thalli to other lichens, often sterile crusts themselves (e.g., members of Lepraria s. str.). While the LSU data showed that the majority of sequences from each amplicon pool were likely to represent Arthoniomycetes, the 454-generated ITS data were not as simple to interpret, with no class identification consistently being associated with the majority of sequences from each sample. There are three potential reasons why this would 
be the case: (1) LSU is more conserved than ITS, making it easier for a classifier to assign a sequence to a class even when close relatives are not present in the database, (2) ITS sequences often have large introns in the SSU portion at the 5' end, the end from which amplicons were sequenced, meaning that reads from certain taxa may not contain any ITS sequence data, and (3) the LSU database contains a member of the family Chrysotricaceae ('Chrysothrix' caesia), which is the most closely related family to Andreiomycetaceae according to phylogenetic analyses, while the ITS database contains only members of distantly related families within Arthoniomycetes/Arthoniales. The Sanger-generated ITS data (for which only partial sequences could be obtained for only half of the samples) support the notion that a long $\sim 0.5 \mathrm{~kb}$ intron is present at the 5' end of the Andreiomyces morozianus amplicon. These ITS sequences show high BLAST-based similarity to Lepraria obtusatica. Analyses of non-molecular character data also show high similarity between L. obtusatica and A. morozianus, supporting the recombination of this additional species into the new genus as Andreiomyces obtusaticus. The species A. obtusaticus differs from A. morozianus only in having a slightly different chemistry and distribution. The morphology and major secondary chemistry (obtusatic acid) of the two species are essentially identical. Although these species were considered likely to be unrelated to the core of the genus Lepraria (Lendemer 2010), the basic morphology alone did not allow a definitive placement elsewhere. Notably, our results for A. obtusaticus are reconcilable with those of Ekman and Tonsberg (2002), where the species was on a relatively long branch and there was not support for its phylogenetic position; due to the sampling in that study, one is not able to evaluate the placement of the species with regard to other members of the class Arthoniomycetes.

The high-throughput procedures used in this publication should prove useful for anyone attempting to target the well-studied fungal LSU and ITS loci for studying the systematics of specific fungi, especially if the organisms of interest cannot be extracted from the other organisms with which they reside in nature. The two-pronged (ITS plus LSU) approach that we use here is ideal for phylogenetic placement. Previous studies that we have conducted on sterile crustose lichen-forming fungi have primarily taken advantage of the ITS region for placing fungi that have ended up belonging to well-established families and genera (Hodkinson \& Lendemer 2012c; Lendemer \& Hodkinson 2012b, 2013). However, in the present case, where the family and genus were yet to be established, LSU was much more effective because of its higher level of conservation. When the goal is to resolve the dispositions of diverse problematic fungi that remain unnamed, incertae sedis, or have taxonomic positions that are not expected to reflect their true phylogeny, one cannot know a priori which locus will be most informative; however, between the two of them, it is likely that placement will be possible, and that one will be able to determine the 'barcode' for the primary fungus and use it for future identifications.

The procedures outlined here could be especially useful for the Barcode of Life effort (Seifert 2009). The goal of this project is to generate sequences from a small number of loci and a large number of individual organisms (ideally, all organisms). For fungi, the ITS region has been established as the 'official' DNA barcode region, with LSU often suggested as a supplemental region due to its more conserved sequence. Our approach could both accelerate barcoding and bring down its cost by providing an alternative to cloning when dealing with samples that have a high level of environmental contamination. This method is especially useful when working with a diverse array of poorly-studied, unculturable organisms, as demonstrated by our work.

\section{Taxonomy}

Andreiomycetaceae Hodkinson \& Lendemer fam. nov.

Mycobank Number: 805806

TYPE GENUS - Andreiomyces Hodkinson \& Lendemer

Diagnosis. - An unusual member of the order Arthoniales characterized by having a coccoid photobiont, persistently sterile thallus, and the production of obtusatic acid together with usnic or isousnic acids. 


\section{Andreiomyces.}

Etymology - The name is formed automatically following the included genus

Andreiomyces Hodkinson \& Lendemer gen. nov.

Mycobank Number: 805807

TYPE SPECIES - Andreiomyces morozianus (Lendemer) Hodkinson \& Lendemer

Diagnosis. - A remarkable lichen genus belonging to the monogeneric family Andreiomycetaceae differing from all other members of the Arthoniales in having a coccoid photobiont, persistently sterile thallus, and the production of obtusatic acid.

Etymology. - The epithet commemorates Dr. Andrei "Andy" Moroz (1982-present), spouse of the second author, whose surname was used to form the specific epithet when the species was originally described in Lepraria. We have elected to use binomial nomenclature to honor Dr. Moroz for his companionship during the second author's fieldwork throughout eastern North America.

Andreiomyces morozianus (Lendemer) Hodkinson \& Lendemer comb. nov. Mycobank Number: 805808

Lepraria moroziana Lendemer, Brittonia, 62(3): 282. 2010. - Type: U.S.A. North Carolina, Haywood Co., Great Smoky Mountains National Park, vicinity of Baxter Creek, trail to summit of Mt. Sterling (2 miles to summit), spruce-fir-birch forest with sparse hemlock, on base of rotting Tsuga, 1370-1760 m., 26 April 2006, J. C. Lendemer et al. 11954 (holotype: NY!)

Description - See Lendemer (2010).

Discussion - Andreiomyces morozianus is known exclusively from the Appalachian Mountains, and is the only North American species with a leprarioid growth form for which obtusatic acid is the major secondary compound. The species also produces isousnic acid and an anthraquinone pigment, unlike the similar European species A. obtusaticus (see below).

Andreiomyces obtusaticus (Tønsberg) Hodkinson \& Lendemer comb. nov.

Mycobank Number: 805809

Lepraria obtusatica Tønsberg, Sommerfeltia, 14: 204. 1992. - Type: NORWAY. Hordaland: Askøy, S of lake Askvatnet, on shaded bark of Corylus under dry, overhanging rock, 14 Jun 1984, T. Tønsberg 8832 (holotype: BG; isotypes: DUKE!, E)

Description - See Tønsberg (1992).

Discussion - Andreiomyces obtusaticus is morphologically identical to A. morozianus. It is also similar chemically, as these two species were the only ones previously placed in the genus Lepraria having obtusatic acid as a major secondary metabolite. However, A. morozianus additionally produces isousnic acid and an anthraquinone pigment, both of which are absent in $A$. obtusaticus. The two species also have different geographic ranges, with A. obtusaticus being known only from Europe, while A. morozianus is known only from the Appalachian Mountains of North America.

\section{Acknowledgements}

We would like to thank Maria Prieto, Mats Wedin, Richard C. Harris, Robert Lücking, Sarah Hodkinson and Jim Lawrey for discussions and assistance. This work was supported by the National Science Foundation under grant DEB-1145511.

\section{References}

Edgar RC. 2010 - Search and clustering orders of magnitude faster than BLAST. Bioinformatics 26(19), 2460-2461.

Edgar RC, Haas BJ, Clemente JC, Quince C, Knight R. 2011 - UCHIME improves sensitivity and speed of chimera detection. Bioinformatics 27(16), 2194-2200. 
Edwards RA, Rodriguez-Brito B, Wegley L, Haynes M, Breitbart M, Peterson DM, Saar MO, Alexander S, Alexander EC, Rohwer F. 2006 - Using pyrosequencing to shed light on deep mine microbial ecology. BMC Genomics 7, 57.

Ekman S, Tønsberg T. 2002 - Most species of Lepraria and Leproloma form a monophyletic group closely related to Stereocaulon. Mycological Research 106(11), 1262-1276.

Ertz D, Tehler A. 2011 - The phylogeny of Arthoniales (Pezizomycotina) inferred from nucLSU and RPB2 sequences. Fungal Diversity 49(1), 47-71.

Gardes M, Bruns TD. 1993 - ITS primers with enhanced specificity for basidiomycetes-application to the identification of mycorrhizae and rusts. Molelcular Ecology 2, 113-118.

Gryganskyi AP, Humber RA, Smith ME, Miadlikowska J, Wu S, Voigt K, Walther G, Anishchenko IM, Vilgalys R. 2012 - Molecular phylogeny of the Entomophthoromycota. Molecular Phylogenetics and Evolution 65(2), 682-694.

Hodkinson BP. 2011 - A phylogenetic, ecological, and functional characterization of nonphotoautotrophic bacteria in the lichen microbiome. Doctoral Dissertation, Duke University, Durham, NC.

Hodkinson BP, Lendemer JC. 2011 - Molecular analyses reveal semi-cryptic species in Xanthoparmelia tasmanica. Bibliotheca Lichenologica 106, 115-126.

Hodkinson BP, Lendemer JC. 2012a - A multi-gene pyrosequencing-based approach for elucidating the phylogeny of diverse lichenized microfungi. In: Mycological Society of America 2012 Meeting. Yale University, New Haven, Connecticut, S1-1450.

Hodkinson BP, Lendemer JC. 2012b - Next-Generation Systematic Botany: A pyrosequencingbased approach for elucidating the phylogeny and informing the taxonomy of sterile crustose lichens. In: Botany 2012: The Next Generation. Botanical Society of America, Columbus, Ohio, abs. 760.

Hodkinson BP, Lendemer JC. 2012c - Phylogeny and taxonomy of an enigmatic sterile lichen. Systematic Botany 37(4), 835-844.

Hodkinson BP, Lutzoni F. 2009 - A microbiotic survey of lichen-associated bacteria reveals a new lineage from the Rhizobiales. Symbiosis 49(3), 163-180.

Hodkinson BP, Lendemer JC, Esslinger TL. 2010 - Parmelia barrenoae, a macrolichen new to North America and Africa. North American Fungi 5(3), 1-5.

Hodkinson, BP, Gottel NR, Schadt CW, Lutzoni F. 2012a - Photoautotrophic symbiont and geography are major factors affecting highly structured and diverse bacterial communities in the lichen microbiome. Environmental Microbiology 14(1), 147-161.

Hodkinson, BP, Uehling JK, Smith ME. 2012b - Lepidostroma vilgalysii, a new basidiolichen from the New World. Mycological Progress, 11(3), 827-833.

Hodkinson, BP, Uehling JK, Smith ME. 2012c - Data from: Lepidostroma vilgalysii, a new basidiolichen from the New World. Dryad Digital Repository. doi:10.5061/dryad.j1g5dh23

Hofstetter V, Miadlikowska J, Kauff F, Lutzoni F. 2007 - Phylogenetic comparison of proteincoding versus ribosomal RNA-coding sequence data: A case study of the Lecanoromycetes (Ascomycota). Molecular Phylogenetics and Evolution 44, 412-426.

James TY, Kauff F, Schoch CL, Matheny PB, Hofstetter V, Cox CJ, Celio G, Gueidan C, Fraker E, Miadlikowska J, Lumbsch HT, Rauhut A, Reeb V, Arnold AE, Amtoft A, Stajich JE, Hosaka K, Sung GH, Johnson D, O'Rourke B, Crockett M, Binder M, Curtis JM, Slot JC, Wang Z, Wilson AW, Schußler A, Longcore JE, O'Donnell K, Mozley-Standridge S, Porter D, Letcher PM, Powell MJ, Taylor JW, White MM, Griffith GW, Davies DR, Humber RA, Morton JB, Sugiyama J, Rossman AY, Rogers JD, Pfister DH, Hewitt D, Hansen K, Hambleton S, Shoemaker RA, Kohlmeyer J, Volkmann-Kohlmeyer B, Spotts RA, Serdani M, Crous PW, Hughes K., Matsuura K, Langer E, Langer G, Untereiner WA, Lucking R, Budel B, Geiser DM, Aptroot A, Diederich P, Schmitt I, Schultz M, Yahr R, Hibbett DS, Lutzoni F, McLaughlin DJ, Spatafora JW, Vilgalys R. 2006 - Reconstructing the early evolution of Fungi using a six-gene phylogeny. Nature. 443, 818-822. 
Katoh K, Misawa K, Kuma KI, Miyata T. 2002 - MAFFT: a novel method for rapid multiple sequence alignment based on fast Fourier transform. Nucleic Acids Res 30, 3059-3066.

Lendemer JC. 2010 - Notes on Lepraria s.1. (Lecanoromycetes, Ascomycota) in North America: New species, new reports, and preliminary keys. Brittonia 62(3), 267-292.

Lendemer JC. 2011 - A standardized morphological terminology and descriptive scheme for Lepraria (Stereocaulaceae). Lichenologist 43, 379-399.

Lendemer JC. 2012 - Lichen taxonomy for the $21^{\text {st }}$ century: a revision of the genus Lepraria s.l. in North America north of Mexico. Doctoral Dissertation, CUNY Lehman Coll., Bronx, NY.

Lendemer JC, Elix JA. 2010 - Two new species of Chrysothrix from eastern North America. Opuscula Philolichenum 8, 51-58.

Lendemer JC, Hodkinson BP. 2009 - The Wisdom of Fools: new molecular and morphological insights into the North American apodetiate species of Cladonia. Opuscula Philolichenum 7, 79-100.

Lendemer JC, Hodkinson BP. 2010 - A new perspective on Punctelia subrudecta in North America: previously-rejected morphological characters corroborate molecular phylogenetic evidence and provide insight into an old problem. The Lichenologist 42(4), 405-421.

Lendemer JC, Hodkinson BP. 2012a - Sterile asexually reproducing crustose lichens: improving classification, conservation, and communication in the 21st century. In: Mycological Society of America 2012 Meeting. Yale University, New Haven, Connecticut, OS7-1445.

Lendemer JC, Hodkinson BP. 2012b - Chirleja buckii, a new genus and species of lichenized fungi from Tierra del Fuego, southern South America. New Zealand Journal of Botany 50(4), 449-456.

Lendemer JC, Hodkinson BP. 2013 - A radical shift in the taxonomy of Lepraria s.l.: molecular and morphological studies shed new light on the evolution of asexuality and lichen growth form diversification. Mycologia 105, 10.3852/12-338.

Lendemer JC, Knudsen K, Elix JA. 2008 - Lepraria friabilis, a new species from eastern North America. Opuscula Philolichenum 5, 61-66.

Ley RE, Turnbaugh PJ, Klein S, Gordon JI 2006 - Microbial ecology: human gut microbes associated with obesity. Nature 444, 1022-1023.

Liu Z, Lozupone C, Hamady M, Bushman FD, Knight R. 2007 - Short pyrosequencing reads suffice for microbial community analysis. Nucleic Acids Research 35, e120.

Maddison WP, Maddison DR. 2010 - Mesquite: a modular system for evolutionary analysis, version 2.74. http://mesquiteproject.org.

Margulies M, Egholm M, Altman WE, Attiya S, Bader JS, Bemben LA, Berka J, Braverman MS, Chen YJ, Chen ZT, Dewell SB, Du L, Fierro JM, Gomes XV, Godwin BC, He W, Helgesen S, Ho CH, Irzyk GP, Jando SC, Alenquer MLI, Jarvie TP, Jirage KB, Kim JB, Knight JR, Lanza JR, Leamon JH, Lefkowitz SM, Lei M, Li J, Lohman KL, Lu H, Makhijani VB, McDade KE, McKenna MP, Myers EW, Nickerson E, Nobile JR, Plant R, Puc BP, Ronan MT, Roth GT, Sarkis GJ, Simons JF, Simpson JW, Srinivasan M, Tartaro KR, Tomasz A, Vogt KA, Volkmer GA, Wang SH, Wang Y, Weiner MP, Yu PG, Begley RF, Rothberg JM. 2005 - Genome sequencing in microfabricated high-density picolitre reactors. Nature 437, 376-380.

Rambaut A. 2009 - FigTree v. 1.3.1. Program distributed by the author. Institute of Evolutionary Biology, University of Edinburgh, Edinburgh, U.K.

Rehner SA, Samuels GJ. 1994 - Taxonomy and phylogeny of Gliocladium analysed from nuclear large subunit ribosomal DNA sequences. Mycol. Res. 98(6), 625-634

Roeding F, Borner J, Kube M, Klages S, Reinhardt R, Burmester T. 2009 - A 454 sequencing approach for large scale phylogenomic analysis of the common emperor scorpion (Pandinus imperator). Molecular Phylogenetics and Evolution 53(3), 826-834.

Roesch LFW, Fulthorpe RR, Riva A, Casella G, Hadwin AKM., Kent AD, Daroub SH, Camargo FAO, Farmerie WG, Triplett EW. 2007 - Pyrosequencing enumerates and contrasts soil microbial diversity. The ISME Journal 1, 283-290. 
Schmull M, Miadlikowska J, Pelzer M, Stocker-Wörgötter E, Hofstetter V, Fraker E, Hodkinson BP, Reeb V, Kukwa M, Lumbsch HT, Kauff F, Lutzoni F. 2011 - Phylogenetic affiliations of members of the heterogeneous lichen-forming genus Lecidea sensu Zahlbruckner (Lecanoromycetes, Ascomycota). Mycologia 103(5), 983-1003.

Schloss PD, Westcott SL, Ryabin T, Hall JR, Hartmann M, Hollister EB. 2009 - Introducing Mothur: open-source, platform-independent, community-supported software for describing and comparing microbial communities. Appl Environ Microbiol 75, 7537-7541.

Schoch, C. L., G.-H. Sung, F. López-Giráldez, J. P. Townsend, J. Miadlikowska, V. Hofstetter, B. Robbertse, P. B. Matheny, F. Kauff, Z. Wang, C. Gueidan, R. M. Andrie, K. Trippe, L. M. Ciufetti, A. Wynns, E. Fraker, B. P. Hodkinson, G. Bonito, R. Yahr, J. Z. Groenewald, M. Arzanlou, G. S. de Hoog, P. W. Crous, D. Hewitt, D. H. Pfister, K. Peterson, M. Gryzenhout, M. J. Wingfield, A. Aptroot, S.-O. Suh, M. Blackwell, D. M. Hillis, G. W. Griffith, L. A. Castlebury, A. Y. Rossman, H. T. Lumbsch, R. Lücking, B. Büdel, A. Rauhut, P. Diederich, D. Ertz, D. M. Geiser, K. Hosaka, P. Inderbitzin, J. Kohlmeyer, B. Volkmann-Kohlmeyer, L. Mostert, K. O’Donnell, H. Sipman, J. D. Rogers, R. A. Shoemaker, J. Sugiyama, R. C. Summerbell, W. Untereiner, P. Johnston, S. Stenroos, A. Zuccaro, P. Dyer, P. Crittenden, M. S. Cole, K. Hansen, J. M. Trappe, F. Lutzoni, and J. W. Spatafora. 2009 - The Ascomycota Tree of Life: A Phylum Wide Phylogeny Clarifies the Origin and Evolution of Fundamental Reproductive and Ecological Traits. Systematic Biology 58(2), 224-239.

Seifert KA. 2009 - Progress towards DNA barcoding of fungi. Molecular Ecology Resources 9, 83-89.

Smith SA., Wilson NG, Goetz FE, Feehery C, Andrade SCS, Rouse GW, Giribet G Dunn CW. 2011 - Resolving the evolutionary relationships of molluscs with phylogenomic tools. Nature 480, 364-367.

Stamatakis A. 2006 - RAxML-VI-HPC: maximum likelihood-based phylogenetic analyses with thousands of taxa and mixed models. Bioinformatics 22, 2688-2690.

Taranger J, Trollfors B, Lind L, Zackrisson G, Beling-Holmquist K. 1994 - Environmental contamination leading to false-positive polymerase chain reaction for pertussis. Pediatr. Infect. Dis. J. 13, 936-937.

Timme RE, Bachvaroff TR, Delwiche CF. 2012 - Broad Phylogenomic Sampling and the Sister Lineage of Land Plants. PLoS ONE 7(1), e29696.

Tønsberg, T. 1992 - The sorediate and isidiate, corticolous, crustose lichens in Norway. Sommerfeltia 14, 1-331.

Turnbaugh PJ, Ley RE, Mahowald MA, Magrini, Mardis ER, Gordon JI. 2006 - An obesityassociated gut microbiome with increased capacity for energy harvest. Nature 444, 1027-1031.

Turnbaugh PJ, Ley RE, Hamady M, Fraser-Liggett CM, Knight R, Gordon JI. 2007 - The Human Microbiome Project. Nature 449, 804-810.

Vilgalys R, Hester M. 1990 - Rapid genetic identification and mapping of enzymatically amplified ribosomal DNA from several Cryptococcus species. J Bacteriol 172, 4238-4246.

White TJ, Bruns TD, Lee S, Taylor J. 1990 - Amplification and direct sequencing of fungal ribosomal RNA genes for phylogenetics. In: Innis, M.A., Gelfand, D.H., Sninsky, J.J. \& White, T.J. (eds.). PCR Protocols: A Guide to Methods and Applications. Pp. 315-322. Academic Press, San Diego.

Zhang Z, Schwartz S, Wagner L, Miller W. 2000 - A greedy algorithm for aligning DNA sequences. Journal of Computational Biology 7, 203-214. 